\title{
Improvement of wireless transmission system performance for EEG signals based on development of scalar quantization
}

\author{
Mohsen Habib Nezhad ${ }^{1}$, Khazaimatol S. Subari ${ }^{1}$, Mehran Yahyavi ${ }^{2,3}$ \\ 1. Faculty of Engineering, Multimedia University, Cyber Jaya, Malaysia \\ 2. Department of Signal Theory and Communications (TSC), Polytechnic University of Catalonia, Barcelona, Spain \\ 3. E-mail any correspondence to: yahyavi.mehran@gmail.com
}

\begin{abstract}
Advancement of wireless technology leads to some developments in current wireless electroencephalography. Through improving the transmission method of brainwaves, it would be possible to bring more convenience for the patients in need and give this opportunity to others for discovering other aspects of the amazing brainwave. What has been proposed in this study is a new type of adjustable backward quantization method which exploits the nature of the brainwave signal. This method is based on the nature of the captured brainwave and its quantization boundary changes based on the amplitude of each EEG captured signal. The proposed quantization scheme has been analyzed with uniform and Gaussian distribution of quantization level. Consequently, the Backward Gaussian Quantization with Adjustable Boundary and two Word Memories beside the Backward Uniform Quantization with Adjustable Boundary and two Word Memories are introduced by this experiment. In addition, the performance of wireless transmission system and the proposed quantizer's efficiency for very low frequency (up to $100 \mathrm{~Hz}$ ) and amplitude EEG signal have been noticed. With doing so, we simulated the transmitter and receiver by MATLAB ${ }^{\circledR}$ software. To model the medium, channel was assumed as Additive White Gaussian Noise (AWGN). Meanwhile analysis is done for the whole wireless system performance in terms of transmission range, compared with current available wireless transmission systems on the market. It should be noticed that the transmission range of the proposed wireless transmission system is compared to the transmission range of current wireless EEG systems when there is no obstacle between transmitter and receiver. Furthermore, some relevant parameters to evaluate the quality of the proposed quantization method were examined. To sum up, the proposed quantization schemes show considerable performance in terms of Quantization Rate for constant MSQE and SQNR in comparison with Uniform Quantization method and the achieved transmission range of our wireless system by using this method is higher than available wireless EEG systems on market.
\end{abstract}

Keywords: Wireless electroencephalogram, adjustable backward quantization, brainwave.

\section{Introduction}

The electroencephalogram, better known as EEG, has the ability to record the activity that takes place in the brain from the brain scalp. Hans Berger (1929) recorded the first brain activity based on studies carried out on animals as early as 1870. Typically a massive recording device has been used in different electroencephalography (EEG) applications. It is connected to the patients via leads and these patients are tied to their beds or seats. With the revolution in wireless telecommunication and manufacturing technology, the invention of the mobile electronic device made its debut in the market. The presence of the current wireless communication that has taken over old wired solutions is presented here in the EEG recorders. Moreover, the process of monitoring the brains' activities wirelessly is not only convenient but also opens up the possibility of measuring the brain activity of moving subjects $[1,24]$.The recorded waveforms reflect the activity that occurs at the cortex, the surface of the brain, and are said to be influenced by the electrical activity from the brain structures located beneath the cortex.

\section{EEG applications}

Generally EEG signal application is divided into three major groups: medical purposes [2]; biometric purposes [3, 20]; and entertainment purposes [2, 20].

In wireless EEG systems, the efficiency of using brainwaves for a specific purpose is strictly dependent on the performance of the wireless system. Undoubtedly, wireless communication gives us more capabilities to utilize EEG signals. However, a corresponding increase in the barriers for transmitting EEG occurs. In the next part, we will briefly discuss existing problems in the current wireless EEG systems [1, 21, 23].

\section{Existing problems in current EEG wireless systems} While the design of wireless systems depends on the intended application of EEG [4, 22], the performance of current wireless EEG systems will be significantly enhanced if some telecommunication design parameters are considered. These parameters include:

1. Transmission range: Most of the current wireless EEG systems suffer from low transmission range, usually less than a few hundred meters. A common method for incrementing the transmission range is increasing power of the output signal which leads to more power consumption $(5,23)$.

2. Output bit rate limitation: According to the bandwidth characteristics of the EEG signal and the Shannon channel capacity formula, there is a limitation for transmitting digital output. The output bit rate of the 
transmitter should not overstep Shannon thresholds. Regarding this threshold, when the bandwidth is constant for a higher bit rate, a higher signal to noise ratio is required $[6,22,23]$.

3. Power consumption: Generally, wireless EEG systems use headsets instead of massive wires. This headset should be able to work with rechargeable batteries, thus requiring the design of wireless EEG systems to consider battery life. Since the power consumption of the components determines the life time of batteries, it is better to use techniques and devices that reduce power consumption without affecting the performance of a wireless system [7, 22]

4. Inaccuracy of received signals: When the previous limitations (stated in the previous paragraphs), are forced in the design of a wireless EEG system, one of the solutions is to reduce the number of bits for presenting each sample. By reducing the number of bits per sample the power consumption decreases. Conversely, when converting the digitalized EEG to analog form at the receiver, an egregious difference between transmitted EEG and received signal is observed [8, 21, 22].

These parameters should be primary considerations in the design of all wireless EEG systems. In contemporary wireless systems these parameters are strictly related to each other. For example, increasing the transmission range and output bit rate of the transmitter leads to increasing power consumption. Reduction of the battery power leads to a decreased transmission range. It is impossible to satisfy all the desired states in design of wireless systems perfectly. So there is trade-off depending on the design of the system [5-8, 21-23].

\section{Motivation of the study}

EEG signals contain frequency components that are typically less than $100 \mathrm{~Hz}$ and amplitudes do not exceed $\pm 100 \mu \mathrm{V}$. Since EEG signals have such a low frequency, signal difference between two consecutive samples' amplitude would not go very far. In this project we design a wireless EEG transceiver to leverage this fact. By anticipating the next possible EEG sample interval it would be possible to develop a quantization method for EEG as a part of a telecommunication system.

In this project, the main priority was to enhance a conventional telecommunication system by advanced wireless technology. Telecommunication parameters have been used to evaluate performance of this project plus relevant parameters to evaluate performance of the proposed quantizer scheme. SNR is one of the most wellknown parameters which are invoked for evaluating different approaches such as EEG at various stages (capturing and transmission) [27].

\section{Current method of scalar quantization}

In this section we briefly introduce some methods of scalar quantization and explain the strengths and weaknesses of these methods when we are dealing with very low frequency and low amplitude.

Generally scalar quantization can be divided into three major groups: uniform quantization, non-uniform quantization, and entropy-code quantization. Each of these groups can also be further subdivided into more groups [9].

\section{Uniform quantization}

A uniform quantizer is the simplest type of quantizer in which quantization intervals have an equal step size, except perhaps for the outer intervals. In other words, the decision boundaries are uniformly spaced. Reconstructed values also have equal space when decision boundaries are even. The constant spacing between decision boundaries is known as the step size and is here denoted by $\Delta$.

Suppose we want to model M-level uniform quantizer for a source where its distribution is uniform within interval $\left[-\mathrm{X}_{\max }, \mathrm{X}_{\max }\right]$. So, the step size $\Delta$ is given by

$$
\Delta=2 X_{\max } / M \text {, }
$$

where $M$ represents the number of levels and $X_{\max }$ shows maximum of absolute values of the input source. The distortion in this case becomes:

$$
\sigma_{q}^{2}=2 \sum_{i=1}^{i=M / 2} \int_{(i-1) \Delta}^{i \Delta}\left(x-\frac{2 i-1}{2} \Delta\right)^{2} \frac{1}{2 x \max } d x
$$

By calculating this integral, we find that the MSQE is $\Delta 2 / 12$ [9].

In the cases that use a fixed length code and each code word is presented by $\mathrm{N}$ bits, the number of code words or the number decision points, $\mathrm{M}$, is $2^{\mathrm{N}}$. The SNQR (signal to quantization noise ratio) is calculated by:

$$
\operatorname{SNQR}(d B)=6.02 \cdot N(d B)
$$

According to Eq. (4) for low amplitude signals, the weaknesses of uniform quantization on digitalizing of EEG are much higher than for high amplitude signal. By using this method there is a high amount of distortion for low amplitude samples.

$$
S N Q R=3 M^{2} \frac{\bar{m}^{2}(t)}{\hat{m}_{p}^{2}}
$$

Eq. (4) shows that SNQR for a low amplitude signal is lower than for a high amplitude signal. According to this Eq.(4), $\bar{m}^{2}(t)$ shows the mean squared value of the signal, which is near to zero, and $\hat{m}_{p}^{2}$ shows the squared of the maximum sample value which can be up to $100 \mu \mathrm{V}$. Therefore, the effect of quantization noise on such EEG signals should be noticeable [10]. However, though simplicity of a uniform quantizer is desired, many sources 
have a non-uniform distribution. In these cases, even if the input sources are bordered, dividing the maximum range of the input source variation into the number of quantization levels and assign a code word to each of them does not produce good results [9]. In fact, EEG signals tend to be such a non-uniform distribution with low amplitudes. Using this scheme for quantization of brainwave signals, one would face a mismatch between source distribution and the distribution of quantization level. The discussed problem leads to an increase in the amount of MSQE and to a decrease in the value of SQNR in quantization systems [9, 10].

Two main approaches exist for adapting the quantizer parameters: the first is off-line, or forward adaptive, and the second is on-line or backward adaptive. In the forward adaptive quantization method, the output of the source is divided into several blocks of data. All blocks are analyzed individually before quantization, and the quantizer parameters are set accordingly. Then the settings of the quantizer are transmitted to the target receiver as side information. In the backward adaptive quantization, the adaptation is accomplished based on the previous sample or the current quantizer output. This information is available on both transmitter and receiver side and there is no need for side information transmission [11].

The first approach for adapting the quantizer's variance to changes in input signal variance forces the use of a delay since it is needed for processing the block of data. The attachment of side information with the transmitted signal also needs synchronization between transmitter and receiver. The synchronization leads to increased receiver complexity. The dimension of the block of processed data also influences a number of other problems. For example if the size of the block of processed data is too large, then the matching process may not capture the variations that happen in the input signal statistics. Furthermore, the larger size of the block of data means more delay, which would not be acceptable in most applications. On the other hand, a smaller size of block of data means that one has to transmit the side information more often, which sequentially leads to an increased amount of overhead per sample. The size of the block of data is a trade-off between the loss of accuracy because of negative effect of large block sizes on variance estimation, and increment in the required side information by small block sizes. Furthermore, in this approach we assumed that the input signal has a mean value equal to zero. The information about estimated variance also requires it to be quantized before transmission. But the number of bits which is needed by the quantizer to quantize the value of the estimated variance is significantly more than the number of bits used to present each sample $[9,12]$.

In the backward adaptive quantization approach, the past quantized samples are accessible for adoption between the quantizer and source. The input sample's values are only known to the quantizer and totally unknown to the dequantizer [9].

\section{Non-uniform quantization}

A direct approach for designing the non-uniform quantizer, when the probability model of the input signal is known, is to find the value of $b_{j}$ and $y_{j}$ to minimize Eq. (5). In order to find that we should set the derivative of Eq. (5) with respect to $y_{j}$ equal to zero, and solving for $y_{j}$.

$$
\sigma_{q}^{2}=\sum_{i=1}^{M} \int_{b j-1}^{b j}\left(x-y_{j}\right)^{2} f_{x}(x) d x
$$

Where $M$ is number of quantization levels, $b$ shows the quantization level's boundary, $x$ indicates original value of source, $y_{j}$ indicates the retrieved signal after quantization and $f_{x}(x)$ indicates the probability density function of the input source.

We get:

$$
y_{j}=\frac{\int_{b j-1}^{b j} x f(x) d x}{\int_{b j-1}^{b j} f(x) d x}
$$

By taking the derivative from Eq. (5) with respect to $b_{j}$ and setting it equal to zero, we can then represent $b_{j}$ by

$$
b_{j}=\left(y_{j}+1+y_{j}\right) / 2
$$

According to the Eq. (7), midpoints of the neighboring reconstruction levels can simply be chosen as decision boundary. By solving these two equations we achieve optimal values for the reconstruction levels and decision points that can minimize the mean squared quantization error. But unfortunately, to solve Eq. (6) we need to know the values of $b_{j}$ and $b_{j}-1$, and to solve the Eq. (7), we need to know the values of $y_{j}+1$ and $y_{j}[9,13]$.

Another approach for designing a non-uniform quantizer is the compounded quantization method. The basic idea behind the compounded quantization is in preferentially making the step size smaller, we can make the interval in which the input lies with high probability large or expand the region in which the input lands with high probability in proportion to the probability with which the input lands in this region.

According to Eq. (8), the amount of the quantizer's distortion depends on the input sequence. However, it also tells us how to be released from this dependency. The Bennett integral is described by:

$$
\sigma_{x}^{2}=\int_{-x \max }^{x \max } x^{2} f(x) d x
$$

Where $x$ and $f(x)$ are the value of the original source and its probability density function. Furthermore,

$$
\sigma_{q}^{2}=\alpha^{2} / 3 M^{2} \cdot \sigma_{x}^{2}
$$

where $\alpha$ is constant and $\mathrm{M}$ is the number of levels. Signal to quantizer noise ratio is calculated by: 


$$
\operatorname{SNQR}(d B)=10 \log _{10}\left(3 M^{2}\right)-20 \log _{10} \alpha
$$

Eq. (10) shows the signal to quantizer noise ratio which can be achieved by this method. We can see that this value is slightly improved compared to the uniform quantization results for the non-uniform and low amplitude signals [9, $14,15]$.

\section{Entropy coded quantization}

Three important tasks when designing a quantizer are finding boundary values, finding reconstruction levels, and assigning the code words.

Previous techniques accomplish the first and the second tasks. In both the performance is measured by the mean squared quantization error. In this part we will try to accomplish the third task to allocate code words to the quantization levels. Note that when variable length codes are used, the complexity of the quantizer will increase. We will survey the recent situation and will briefly describe the variable length code assigning [9, 14].

The variable length code assigning to the quantizer outputs can be subdivided into two approaches. A quantizer can be designed by taking into account these facts that influence the rate by selecting the decision boundaries or by leaving the quantizer unchanged, only entropy coding the output of the quantizer.

\section{Methodology}

The distribution of captured EEG signals for each electrode is non-uniform [19]. Tong, et al. [28] have been working on statistical behavior of EEG signal for asphyxic cardiac arrest injury.

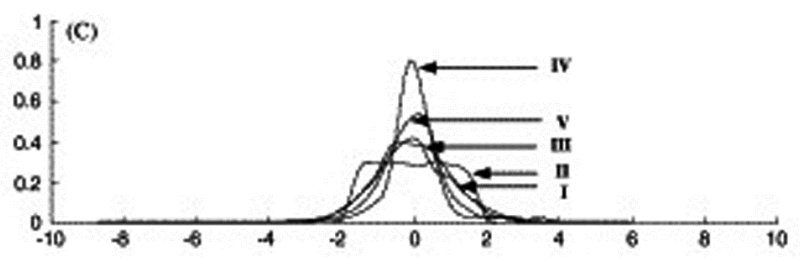

Fig. 1: Examples of captured EEG distribution.

Fig. 1 shows the probability density function of an EEG signal and the $\mathrm{X}$-axis indicates the amplitude of the captured EEG signal that is on the order of microvolts. Since the captured EEG signals are very low frequency, the difference between two consecutive samples is not large. EEG contains low frequency components that decay as a function of frequency. To better express the behavior of the EEG signal in the time domain, a series of cosine function with different coefficient and frequency area is a good way. Generally they could be expressed by:

$$
A(t)=\sum a \cos (2 \pi f t)
$$

The amplitude of the captured EEG can be estimated by substituting real values to the variables. As is required, the rate of captured EEG needs to be at least two times greater than maximum EEG frequency [27]. Deference between two consecutive EEG signals can be generally presented by a combination of cosine waves [16].

$$
\begin{array}{r}
D=A\left(t_{1}\right)-A\left(t_{2}\right)= \\
=\sum a_{1} \cos (\omega 1) \\
-\sum a_{2} \cos (\omega 2)
\end{array}
$$

For frequencies below $50 \mathrm{~Hz}$ and a sampling rate equal to 256 the maximum variation between two consecutive signals is very small. If $f=50 \mathrm{~Hz}, t_{1}=0$, and $t_{2}=1 / 256$, the maximum difference is around:

$$
\begin{array}{r}
D=A\left(t_{1}\right)-A\left(t_{2}\right) \rightarrow \\
\quad a-0.33 a=0.67 a
\end{array}
$$

The point is that, based on the nature of the EEG signal, the amplitude of EEG signals decay with increasing frequency. So if we assume each sample contains all frequency components, the amplitude difference between two consecutive captured EEG samples is equal to subtraction of their amplitudes. This fact simply shows that to design a quantizer, it is not necessary to allocate fixed decision boundaries and since variation of two tandem samples is small, it would be possible to estimate a possible variation area for each EEG sample. This introduces a new approach in design of a quantizer with adjustable quantization interval for each sample and instead of using the old algorithms.

\section{Comprehensive view of the proposed quantization and dequantization algorithm}

Based on the distribution of the EEG signals in each electrode, a quantization scheme with an adjustable quantization boundary would appear to be a suitable solution for solving the existing problems in quantizer design for very low frequency signals. It is shown by the quantization system process which is used in both proposed quantizers in this project, while fig. 2 shows the dequantization process at the receiver side.

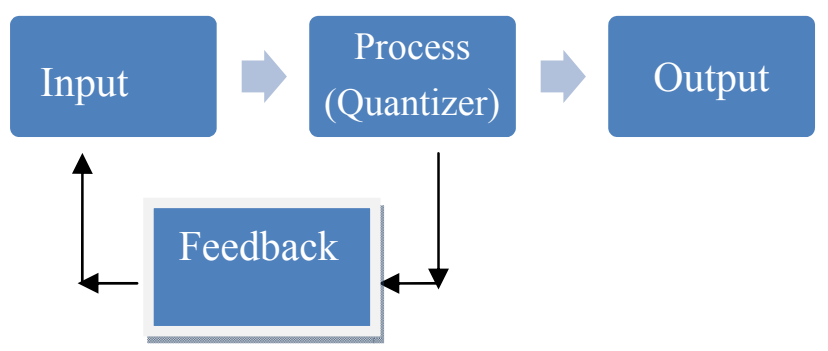

Fig. 2: Simple model of the quantization process. 
According to the fig. 2, the input of the system has feedback from the process box. It means that in this approach, the features of the past input signal which was entered to the quantizer affect the processing procedure of the current input samples. In this study we use the previous sample to approximate the quantization boundaries. This approximation must be same within the boundaries of the dequantizer.

Accordingly in fig. 3, the output of the system is fed back to the process box. In this approach, the past output signal that entered into the dequantizer affect the processing procedure of the current input samples.

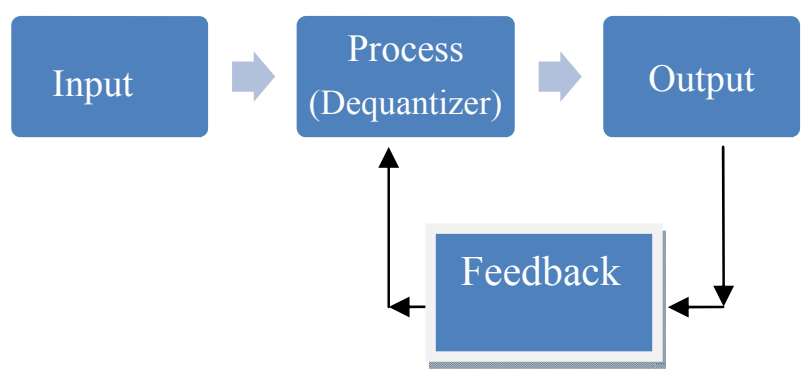

Fig. 3: Simple model of dequantization process.

Note that the input sample that receives feedback from the processing box in the quantizer corresponds with the output sample that is fed back to the processing box in the dequantizer.

\section{Backward Gaussian quantization with adjustable intervals and two word memories (BGAI quantizer with two word memories)}

In order to quantize input signals, we need to find the next possible interval of occurrence. In doing so, we assume each quantization interval includes a mean value and a variance value in which this parameter will change for each sample. The first step to reach this goal is assigning a mean value to our Gaussian distribution. The real mean value of an EEG signal is unknown for the quantizer so we must assume this value. But the assumption should be rational and can cover all the valid interval of occurrence for the EEG signal. So the mean value is assumed zero $(\mu=0)$ and variance is selected to be very large (normally all the valid interval of the EEG variations). This assumed distribution is used for quantizing the first input sample.

In the second step, after entering the first signal to the quantizer, summation of amplitude of the signal and mean of the Gaussian function of the quantizer separated by two results in the mean of a Gaussian distribution quantizer of the next sample. The process is the same for each of the next signals. We create the Gaussian quantization level distribution for each sample by using previous sample feature. According to the estimated mean of the samples in each step, the variance is equal to square of deviation of estimated mean value and previous signal from half of the valid interval for EEG signals. The estimated mean value and entered signal will be saved in the memory for the next sample quantization.

\section{Backward Gaussian dequantization with adjustable intervals and two word memories (BGAI dequantizer with two word memories)}

The initial value of the Gaussian quantization distribution of the quantizer and the dequantizer is the same. So, the first received symbol will be dequantized when it enters into the dequantizer by the initial value. Then, the dequantized signal with initial value is summed with the mean $(\mu=0)$ and separated by two through the dequantizer, which results in a mean of Gaussian distribution dequantizer of the next symbol. According to the mean of the symbols in each time, variance is equal to the square of the deviation of estimated mean from the previous received sample and valid interval for EEG signals. The value of the estimated mean and previous dequantized sample in each stage is saved in the memory for the next sample quantization. Consequently, based on this method, the Gaussian variation of the quantization level in the quantizer is tracked by the dequantizer.

\section{Backward uniform quantization with adjustable intervals and two word memories (BUAI quantizer with two word memories)}

Based on the previous parts, we know this quantization method following the backward quantization idea. It memorizes some information about the characteristics of the previous signal to exploit it for the next samples. In order to quantize input signals, we need to find the mean value of the probability density function distribution. The first step in order to reach this goal is assigning an initial value for mean and variance. These initial values are used for quantizing the first input sample. As with the previous quantizer, here the mean value is assumed zero $(\mu=0)$ and variance is selected to be very large (normally all the valid interval of EEG variations). In this method the quantization levels within the estimated interval are distributed uniformly.

After entering the first signal to the quantizer, summation of amplitude of the signal and assigned mean value of quantizer separated by two, results in the mean of the quantizer of the next sample. Accordingly, the process is the same for the next signals. Based on the mean of the samples in each step, variance is equal to square of deviation of estimated mean and previous signal from half of the valid interval for EEG signals. The estimated mean value and entered signal will be saved in the memory for next sample quantization. Thus we create the new interval for distributing of quantization level for each sample by using previous sample features. 
Backward uniform dequantization with adjustable intervals and two word memories (BUAI dequantizer with two word memories)

The initial value for quantization distribution of the quantizer and dequantizer is the same. So, first symbols will be dequantized when they enter into the dequantizer by the initial value. Then, the dequantized initial value is summed with the mean $(\mu=0)$ and separated by two through the dequantizer, which results in the mean of distribution dequantizer of next symbol. According to the mean in each step, variance is equal to the square of minimum deviation of the estimated mean from previous received sample and half of the valid interval for the EEG signals. The value of the estimated mean and previous dequantized sample in each stage is saved in the memory for next sample quantization. Consequently, based on this method, the variation of quantizion level around the mean value in the quantizer is tracked by the dequantizer.

\section{Results}

This project is a part of large project in the Department of Engineering of the Multimedia University. EEG signals were recorded using a gMobilab + console by Guger Technologies that was connected to a laptop and captured using the MATLAB $^{\circledR}$ Data Acquisition Toolbox with a sampling frequency of $256 \mathrm{~Hz}$. Eight electrodes were placed on the scalp at positions $\mathrm{FC} 3$ and $\mathrm{CP} 3, \mathrm{P} 1$ and P5, FC4 and CP6 and P2 and P6 to record the bipolar EEG signal at pointsC3, $\mathrm{P} 3, \mathrm{C} 4$ and $\mathrm{P} 4$, respectively. The electrodes were placed according to the standard 10-20 international system. Fig.3 shows the position of the electrode placement for the bipolar EEG recording [19].

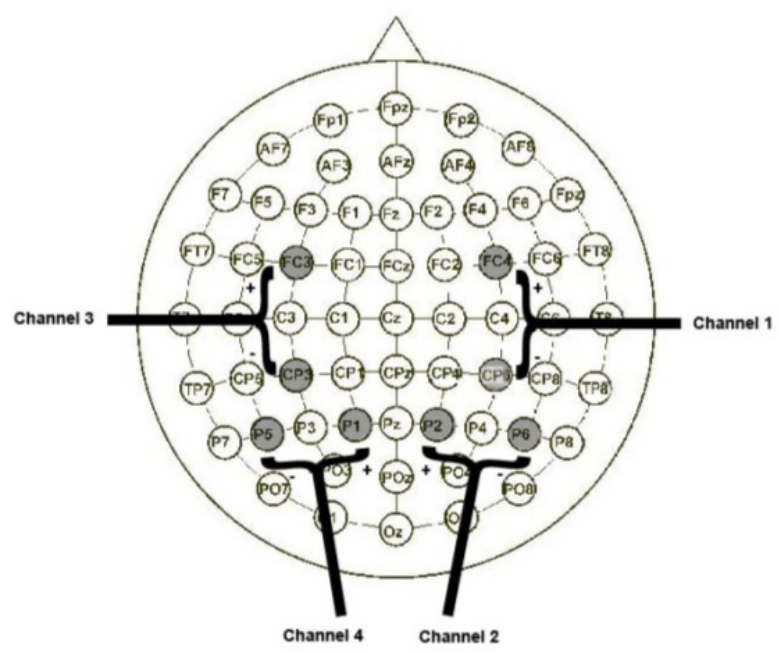

Fig. 4: Position of electrode placements for bipolar EEG recording.

Data were collected from 10 subjects, in 5 separate recording sessions over a course of 2 weeks. All subjects were male students from the Faculty of Engineering, Multimedia University, whose age ranged from 22 to 28 years old. Subjects were required to sit on a reclining chair and remain calm and relaxed throughout the whole recording procedure. They were also required to minimize any movements to avoid any contamination to the EEG signal. During signal acquisition, subjects were asked to clear their minds of any thoughts and relax [19].

Based on the previous discussion we focused on the nature and the time domain behavior brain wave in order to show the characteristics of the EEG signal which by relying on them, we enable to change the decision point appropriately. For this propose, first we found the distribution of the EEG signals captured by each electrode. Note that in this study for finding the distribution of the captured EEG, we have divided the possible EEG variation range in to $12 \times 10^{5}$ segments. Thereafter we have analyzed the number of occurrence of EEG in each segment. The following results are the simulation results that represent the distribution of captured signals for each electrode.

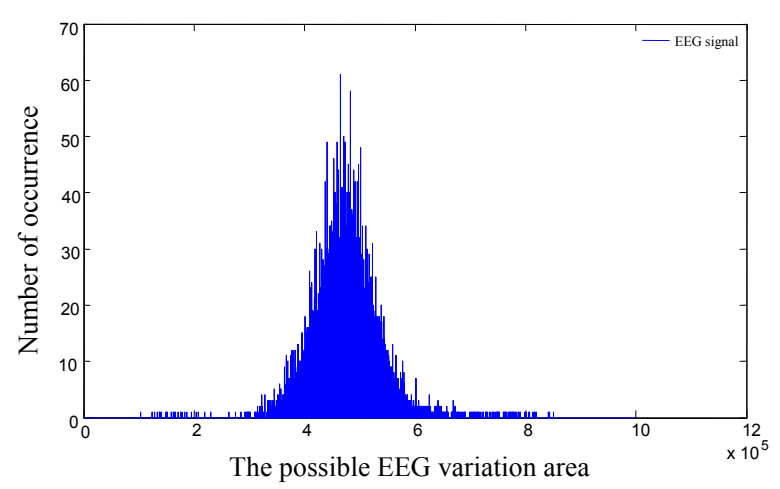

Fig. 5: Histograms of EEG samples captured by channel 1 .

Fig. 5 shows MATLAB ${ }^{\circledR}$ simulation results for histograms of captured EEG signal by electrode 1 . The $\mathrm{X}$-axis indicates the absolute value of the EEG amplitude and the Y-axis indicates the number of occurrences of them.

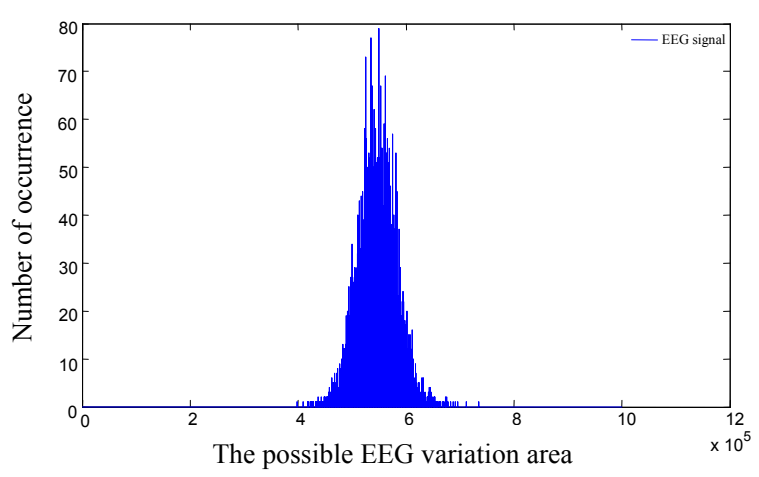

Fig. 6: Histograms of EEG sample captured by channel 2.

Fig. 6 shows MATLAB ${ }^{\circledR}$ simulation results for histograms of captured EEG signal electrode 2.The $\mathrm{X}$-axis indicates absolute value of the EEG amplitude and the $\mathrm{Y}$-axis indicates the number of occurrences of them. 


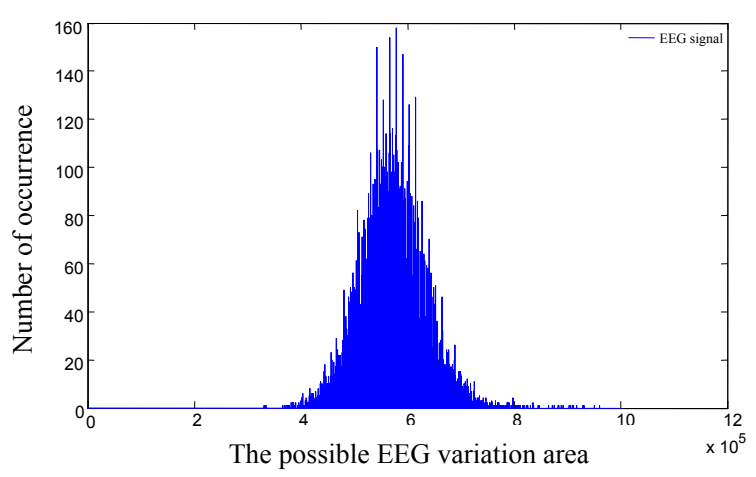

Fig. 7: Histograms of EEG sample captured by channel 3.

Fig. 7 shows MATLAB ${ }^{\circledR}$ simulation results for histograms of captured EEG signals by electrode 3. The $\mathrm{X}$-axis indicates the absolute value of the EEG amplitude and the $\mathrm{Y}$-axis indicates the number of occurrence of them.

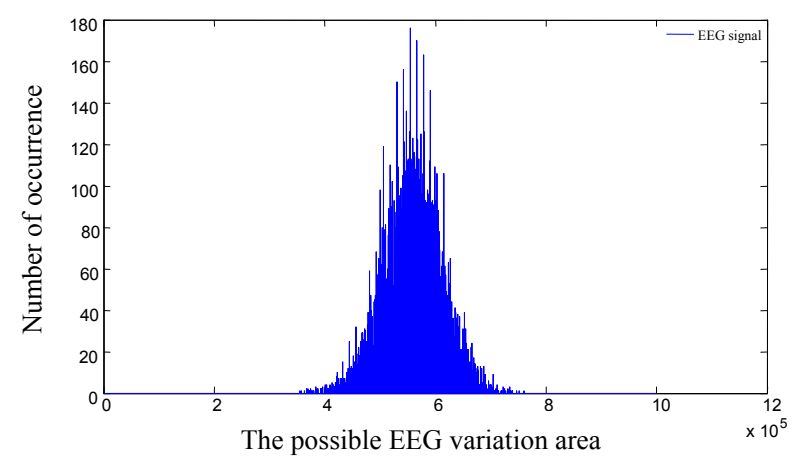

Fig. 8: Histograms of EEG sample captured by channel 4.

Fig. 8 shows MATLAB ${ }^{\circledR}$ simulation results for histograms of captured EEG signals by electrode 4. The X-axis indicates the absolute value of the EEG amplitude and the $\mathrm{Y}$-axis indicates the number of occurrences of them. The results from figs. 5-8 and other surveys on the statistical behavior of the captured EEG show that EEG signal variation around mean is small and in none of the electrodes, the deviation covers all the possible EEG variation area in the duration of recording. So it is not necessary to waste quantization level by assigning decision point to the area which is very far from mean value.

According to the previous discussion about backward Gaussian quantizers with an adjustable boundary and twoword memory, we know that this quantizer is able to estimate the interval whose probability of occurrence in the next sample is very high. Generally no quantizer knows about the next sample that will enter the quantizer, but with the help of this technique and the nature of the EEG signal, we can find an interval in which the next sample definitely is a member. The estimated interval is significantly smaller than the whole range covered by the EEG signal. The quantizer works with this small interval and distributes the quantization levels in this area. Based on the previous discussion the distribution is in Gaussian form. After quantization of each sample, the real value of the sample and estimated mean value will be stored in the memory for the next sample. For all the samples the process is recurred.

Based on them, the mean value of the previous sample and previous sample, we can calculate the variance value for the quantizer for each sample. It is noticeable that all of this estimation is accurate when capturing EEG in a normal situation. It means that if the device that acquires and records the brainwave does not work appropriately for any reason because the characteristics of the EEG signal are not dominant in the recorded signal, the outcome of the quantizer does not follow the variation of the captured EEG signal. In addition for better evaluation of proposed quantization method, we have simulated and designed the whole wireless transmission system.

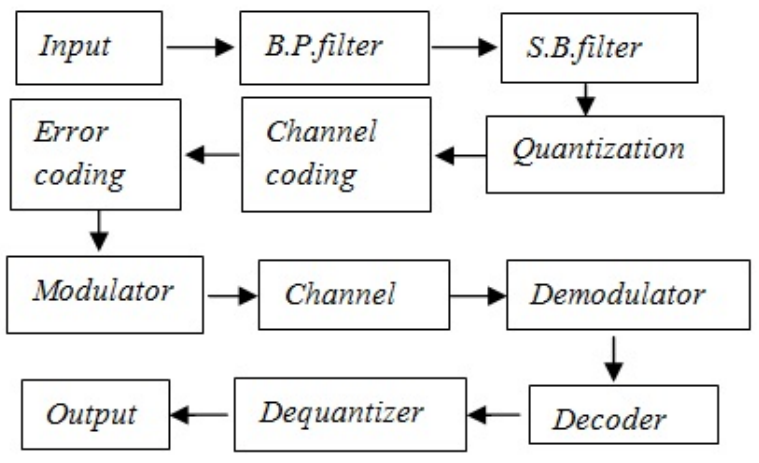

Fig. 9: Block diagram of the simulated transceiver.

Fig. 9 shows the designed transceiver system's structures. Note that in simulation of this model we have used Turbo code as our error detection and correction technique and applied modulation in this study is QPSK. Furthermore, we assume that our channel is AWGN channel and following results come out in these criteria.

\section{Discussion}

Different aspects of brain behaviors are still unknown, and there are so many unanswered questions about known parts of brain behaviors. So far comprehensive investigation has not been done on brainwaves. Researchers for quantization of EEG signals usually had to use the traditional methods developed for other purposes such as sound, speech, and video quantization. In this part, we will compare our results with uniform quantizer outcomes. It is worth noting that uniform quantization currently is used in electroencephalography devices.

Let us calculate the signal to noise ratio (SNR) for this case. The signal variance $\sigma_{\mathrm{s}}{ }^{2}$ for a Gaussian random variable, which takes on values in the interval $\left[-\mathrm{X}_{\max }, \mathrm{X}_{\max }\right]$ is $(10)$ :

$$
\begin{array}{r}
\sigma_{s}{ }^{2}=\left(2 \cdot X_{\max }\right)^{2}= \\
\left(2 \cdot 8 \cdot 10^{-5}\right)^{2}=2.5600 \cdot 10^{-8} \\
S N R=10 \log \left(\sigma_{s}{ }^{2} / \sigma_{q}{ }^{2}\right),
\end{array}
$$


where $\left(\sigma_{\mathrm{q}}^{2}\right)$ is Mean Squared Quantization Error.

\begin{tabular}{cccc}
\hline $\begin{array}{c}\text { Bits per } \\
\text { sample }\end{array}$ & $\begin{array}{c}\text { Uniform } \\
\text { Quantizer } \\
\text { (MSQE) }\end{array}$ & $\begin{array}{c}\text { BGAI } \\
\text { Quantizer } \\
\text { MSQE }\end{array}$ & $\begin{array}{c}\text { BUAI } \\
\text { Quantizer } \\
\text { MSQE }\end{array}$ \\
\hline $\mathbf{5}$ & $-104.29 \mathrm{~dB}$ & $-109.86 \mathrm{~dB}$ & $-118.64 \mathrm{~dB}$ \\
$\mathbf{6}$ & $-110.44 \mathrm{~dB}$ & $-118.26 \mathrm{~dB}$ & $-124.98 \mathrm{~dB}$ \\
$\mathbf{7}$ & $-116.61 \mathrm{~dB}$ & $-125.68 \mathrm{~dB}$ & $-131.14 \mathrm{~dB}$ \\
$\mathbf{8}$ & $-122.62 \mathrm{~dB}$ & $-131.69 \mathrm{~dB}$ & $-137.21 \mathrm{~dB}$ \\
$\mathbf{9}$ & $-128.65 \mathrm{~dB}$ & $-136.39 \mathrm{~dB}$ & $-143.28 \mathrm{~dB}$ \\
$\mathbf{1 0}$ & $-134.66 \mathrm{~dB}$ & $-142.84 \mathrm{~dB}$ & $-149.28 \mathrm{~dB}$ \\
$\mathbf{1 1}$ & $-140.72 \mathrm{~dB}$ & $-149.19 \mathrm{~dB}$ & $-155.24 \mathrm{~dB}$ \\
$\mathbf{1 2}$ & $-146.70 \mathrm{~dB}$ & $-155.67 \mathrm{~dB}$ & $-161.22 \mathrm{~dB}$ \\
$\mathbf{1 3}$ & $-152.76 \mathrm{~dB}$ & $-160.95 \mathrm{~dB}$ & $-167.27 \mathrm{~dB}$ \\
$\mathbf{1 4}$ & $-158.73 \mathrm{~dB}$ & $-166.65 \mathrm{~dB}$ & $-173.32 \mathrm{~dB}$ \\
$\mathbf{1 5}$ & $-164.78 \mathrm{~dB}$ & $-172.85 \mathrm{~dB}$ & $-179.37 \mathrm{~dB}$ \\
$\mathbf{1 6}$ & $-170.79 \mathrm{~dB}$ & $-185.00 \mathrm{~dB}$ & $-185.42 \mathrm{~dB}$ \\
$\mathbf{1 7}$ & $-176.80 \mathrm{~dB}$ & $-194.03 \mathrm{~dB}$ & $-191.47 \mathrm{~dB}$ \\
$\mathbf{1 8}$ & $-182.87 \mathrm{~dB}$ & $-200.19 \mathrm{~dB}$ & $-197.55 \mathrm{~dB}$ \\
$\mathbf{1 9}$ & $-188.90 \mathrm{~dB}$ & $-206.25 \mathrm{~dB}$ & $-203.49 \mathrm{~dB}$ \\
$\mathbf{2 0}$ & $-194.88 \mathrm{~dB}$ & $-212.12 \mathrm{~dB}$ & $-209.48 \mathrm{~dB}$ \\
\hline Table 1: The comparison among calculated MSQE of proposed \\
methods and uniform quantizer.
\end{tabular}

Table 1 shows estimated MSQE for different number of bits per samples, $5<\mathrm{N}<20$, of a uniform quantizer, a BGAI quantizer and a BUIA quantizer.

In case of a uniform input signal the MSQE of the uniform quantizer is calculated by this formula [10]:

$$
M S Q E_{\text {uniform }}=\Delta^{2} / 12 \text {, }
$$

where the delta parameter, $\Delta$, is the assigned step size in the uniform quantization.

Table 1 shows the value of MSQE for the quantization method. Both of the proposed quantizers present better performance compared to uniform quantization. Differences between the BGAI quantizer with two-word memories and uniform quantizer increases with an increasing number of bits per sample. According to this table for $\mathrm{N}$ equal to sixteen bits per sample the difference is around $15 \mathrm{~dB}$ which is a considerable value. By using this method we can achieve a certain MSQE with 1 to 3 bits per sample less than for a uniform quantizer. Thus, this quantizer works very well when the accuracy is our priority in the received signal.

According to table 1, for $\mathrm{N}$ smaller than sixteen bits per sample, the performance of a BUAI with two word memories in terms of MSQE is better than the others. So for certain MSQE, it approximately needs one bit per sample less than the BGAI quantizer with two word memories and two bits per sample less than the uniform quantizer. When $\mathrm{N}$ is greater than sixteen bits per sample, the BGAI quantizer with two word memories shows better performance and it approximately needs one bit per sample less than the BUAI quantizer with two word memories and 3 bits per sample less than the uniform quantizer for certain MSQE.
The relationship between SNR or MSQE and the number of bits per sample in the BGAI quantization model is not linear. The MSQE has nonlinear behavior because of Gaussian distribution of the quantization level and based on Eq. 15, the SNR is influenced by the MSQE. According to our observation the MSQE and SNR for this kind of quantizer has small dependency to the input signal. Based on the previous chapters, captured EEG from different parts of the brain has totally different and unpredictable Gaussian distribution.

\begin{tabular}{cccc}
\hline $\begin{array}{c}\text { Bits per } \\
\text { sample }\end{array}$ & $\begin{array}{c}\text { Uniform } \\
\text { Quantizer } \\
\text { (SNR) }\end{array}$ & $\begin{array}{c}\text { BGAI } \\
\text { Quantizer } \\
\text { (SNR) }\end{array}$ & $\begin{array}{c}\text { BUAI } \\
\text { Quantizer } \\
\text { (SNR) }\end{array}$ \\
\hline $\mathbf{5}$ & $28.38 \mathrm{~dB}$ & $33.95 \mathrm{~dB}$ & $42.72 \mathrm{~dB}$ \\
$\mathbf{6}$ & $34.52 \mathrm{~dB}$ & $42.34 \mathrm{~dB}$ & $49.06 \mathrm{~dB}$ \\
$\mathbf{7}$ & $40.69 \mathrm{~dB}$ & $49.77 \mathrm{~dB}$ & $55.22 \mathrm{~dB}$ \\
$\mathbf{8}$ & $46.70 \mathrm{~dB}$ & $55.77 \mathrm{~dB}$ & $61.29 \mathrm{~dB}$ \\
$\mathbf{9}$ & $52.73 \mathrm{~dB}$ & $60.47 \mathrm{~dB}$ & $67.37 \mathrm{~dB}$ \\
$\mathbf{1 0}$ & $58.74 \mathrm{~dB}$ & $66.92 \mathrm{~dB}$ & $73.37 \mathrm{~dB}$ \\
$\mathbf{1 1}$ & $64.80 \mathrm{~dB}$ & $73.28 \mathrm{~dB}$ & $79.33 \mathrm{~dB}$ \\
$\mathbf{1 2}$ & $70.79 \mathrm{~dB}$ & $79.75 \mathrm{~dB}$ & $85.30 \mathrm{~dB}$ \\
$\mathbf{1 3}$ & $76.84 \mathrm{~dB}$ & $85.04 \mathrm{~dB}$ & $91.35 \mathrm{~dB}$ \\
$\mathbf{1 4}$ & $82.86 \mathrm{~dB}$ & $90.73 \mathrm{~dB}$ & $97.40 \mathrm{~dB}$ \\
$\mathbf{1 5}$ & $88.86 \mathrm{~dB}$ & $96.93 \mathrm{~dB}$ & $103.38 \mathrm{~dB}$ \\
$\mathbf{1 6}$ & $94.87 \mathrm{~dB}$ & $109.09 \mathrm{~dB}$ & $109.51 \mathrm{~dB}$ \\
$\mathbf{1 7}$ & $100.88 \mathrm{~dB}$ & $118.11 \mathrm{~dB}$ & $115.56 \mathrm{~dB}$ \\
$\mathbf{1 8}$ & $106.95 \mathrm{~dB}$ & $124.27 \mathrm{~dB}$ & $121.63 \mathrm{~dB}$ \\
$\mathbf{1 9}$ & $112.98 \mathrm{~dB}$ & $130.34 \mathrm{~dB}$ & $127.57 \mathrm{~dB}$ \\
$\mathbf{2 0}$ & $118.96 \mathrm{~dB}$ & $136.20 \mathrm{~dB}$ & $133.56 \mathrm{~dB}$ \\
\hline
\end{tabular}

Table 2: The comparison among calculated SNR of the proposed methods and a uniform quantizer.

Table 2 shows the estimated SNR for different amount of quantization rate, $5<\mathrm{N}<20$, for a uniform quantizer, a BGAI quantizer and a BUIA quantizer. The SNR value is calculated by Eq. (15).

As we know for uniform quantization when the input signal has uniform distribution the signal to noise ratio for the quantizer is calculated by Eq. (3) [9].

But for this case the input signal has Gaussian distribution and the value of the signal to noise ratio is estimated by Eq. (15). The calculated SNR by this equation is smaller than Eq. (3) which is predictable. When the distribution of the quantization error is not matched with the input distribution, the MSQE is increased and as result the SNR is reduced.

Both proposed methods have significant performance in terms of SNR compared to the uniform quantizer. For N smaller than sixteen bits per sample BUAI quantizers with two word memories have better MSQE, so the value of SNR for this method is higher than the other discussed methods. For $\mathrm{N}$ more than sixteen bits per sample, BGAI quantizers with two word memories show higher performance in comparison with other methods. For $\mathrm{N}$ greater than sixteen bits per sample the MSQE for BGAI quantizer with two word memories rapidly reduce and this phenomenon affect the value of the SNR for this quantizer and increases it rapidly. 
We are using the AWGN channel which means that we just consider the effect of white noise on the transmitted signal.

Fig. 10 shows the simulation results of bit error rate for the received signal with signal to noise ratio between $-2 \mathrm{~dB}$ to $2 \mathrm{~dB}$. There are 5 lines with different color which correspond to the number of iterations in the error decoding process. Results show that for SNR equal to $2 \mathrm{~dB}$ we achieve Bit Error Rate $(B E R)=3.5 \times 10^{-5}$ or maximum 4 bits error in $10^{5}$ bits.

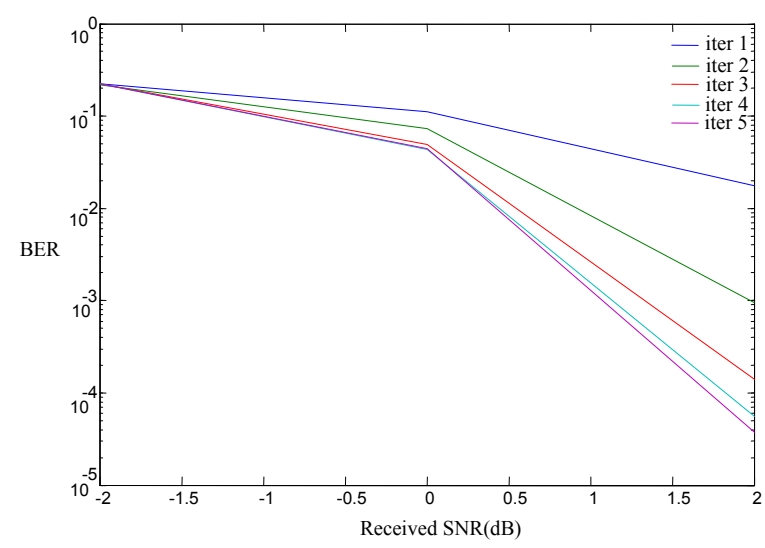

Fig. 10: The achieved BER for received SNR in $(-2 \mathrm{~dB}, 2 \mathrm{~dB})$ intervals in different iterations.

Now if we assume that the minimum SNR of the received signal is $2 \mathrm{~dB}$ we can find the reliable transmission distance between transmitter and receiver. For this propose we used Friis free space Eq. (17). This model can be used when transmitter and receiver has unobstructed line of sight path between them [17].

$$
\operatorname{Pr}(d)=\frac{P t \cdot G t \cdot G r \cdot \lambda^{2}}{(4 \pi)^{2} \cdot d^{2} \cdot L}
$$

where $P t$ presents the transmitter' signal power, $G t$ and $G r$ present transmitter and receiver's antenna gain, in this case the antenna gains are assumed to have unity gain, $L$ presents system loss factor that in case of free space propagation model is equal to $1, d$ represents transmitterreceiver separation, and $\lambda$ presents wavelength of the transmitted signal [17].

In this study we assume the $\lambda$ is equal to one meter which means the system works at $300 \mathrm{MHz}$. Based on application of this system we can choose a lower frequency band. According to Eq. (17), higher value of the wavelength leads to increasing transmission range.

\begin{tabular}{cccc}
\hline $\begin{array}{c}\text { Bits per } \\
\text { sample }\end{array}$ & $\begin{array}{c}\text { Minimum } \\
\text { received SNR }\end{array}$ & $\begin{array}{c}\text { SNR of } \\
\text { transmitted } \\
\text { signal }\end{array}$ & $\begin{array}{c}\text { Transmission } \\
\text { range for } \boldsymbol{\lambda}=\mathbf{1 ~} \mathbf{~ m}\end{array}$ \\
\hline $\mathbf{5}$ & $2 \mathrm{~dB}$ & $42.72 \mathrm{~dB}$ & $8.62 \mathrm{~m}$ \\
$\mathbf{6}$ & $2 \mathrm{~dB}$ & $49.06 \mathrm{~dB}$ & $17.90 \mathrm{~m}$ \\
$\mathbf{7}$ & $2 \mathrm{~dB}$ & $55.22 \mathrm{~dB}$ & $36.39 \mathrm{~m}$ \\
$\mathbf{8}$ & $2 \mathrm{~dB}$ & $61.29 \mathrm{~dB}$ & $73.19 \mathrm{~m}$ \\
$\mathbf{9}$ & $2 \mathrm{~dB}$ & $67.37 \mathrm{~dB}$ & $147.40 \mathrm{~m}$ \\
$\mathbf{1 0}$ & $2 \mathrm{~dB}$ & $73.37 \mathrm{~dB}$ & $281.83 \mathrm{~m}$ \\
\hline
\end{tabular}

Table 3: The transmission range for $\lambda=1$ meter.
Table 3 indicates the value of the transmission range for a BUAI quantizer for $\mathrm{N}$ smaller than eleven. Note that within interval, $4<$ quantization rate $<11$, the output bit rate of the transmitter is less than the maximum channel capacity bit rate which is determined by the Shannon channel theory. Beyond that interval, according to this formula, BER increases enormously.

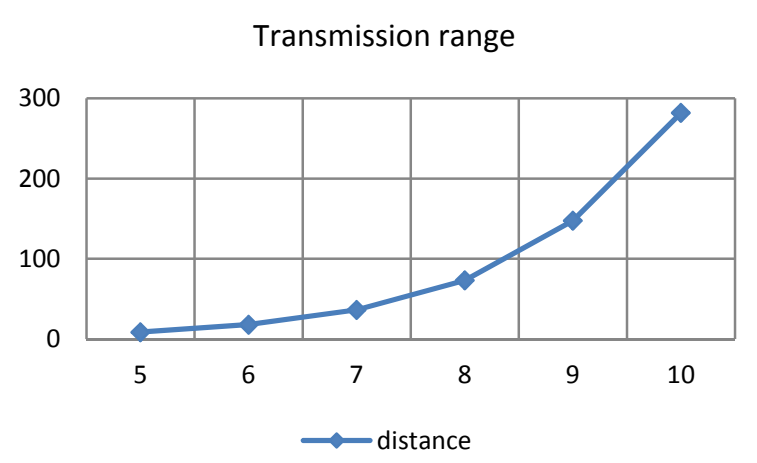

Fig. 11: The transmission range for $\lambda=1$ meter.

Fig. 11 also shows the achieved distance for reliable communication by the BUAI quantizer for different number of quantization rate. In this study we did not apply any amplification before transmission, however for long distance transmission we have to amplify the output signal by increasing the power of the carrier signal.

If value of the $\lambda$ promote to two meter the transmission range in previous figure will promote to four times more than before and when the $\lambda$ becomes four meter the transmission range will become sixteen times more than before.

\begin{tabular}{crrrrr}
\hline $\begin{array}{c}\text { Bits per } \\
\text { sample }\end{array}$ & $\begin{array}{c}\text { Transm. } \\
\text { range } \\
\boldsymbol{\lambda}=\mathbf{0 . 3 3 m}\end{array}$ & $\begin{array}{c}\text { Transm. } \\
\text { range } \\
\boldsymbol{\lambda}=\mathbf{1} \mathbf{~ m}\end{array}$ & $\begin{array}{c}\text { Transm. } \\
\text { range } \\
\boldsymbol{\lambda}=\mathbf{2} \mathbf{~ m}\end{array}$ & $\begin{array}{c}\text { Transm. } \\
\text { range } \\
\boldsymbol{\lambda}=\mathbf{3} \mathbf{~ m}\end{array}$ & $\begin{array}{c}\text { Transm. } \\
\text { range } \\
\boldsymbol{\lambda}=\mathbf{4} \mathbf{~ m}\end{array}$ \\
\hline $\mathbf{5}$ & $0.95 \mathrm{~m}$ & $8.62 \mathrm{~m}$ & $34.48 \mathrm{~m}$ & $77.58 \mathrm{~m}$ & $137.92 \mathrm{~m}$ \\
$\mathbf{6}$ & $1.98 \mathrm{~m}$ & $17.90 \mathrm{~m}$ & $71.6 \mathrm{~m}$ & $161.11 \mathrm{~m}$ & $286.4 \mathrm{~m}$ \\
$\mathbf{7}$ & $4.04 \mathrm{~m}$ & $36.39 \mathrm{~m}$ & $145.56 \mathrm{~m}$ & $327.51 \mathrm{~m}$ & $582.24 \mathrm{~m}$ \\
$\mathbf{8}$ & $8.15 \mathrm{~m}$ & $73.19 \mathrm{~m}$ & $292.76 \mathrm{~m}$ & $658.71 \mathrm{~m}$ & $1171.04 \mathrm{~m}$ \\
$\mathbf{9}$ & $16.37 \mathrm{~m}$ & $147.40 \mathrm{~m}$ & $589.6 \mathrm{~m}$ & $1326.6 \mathrm{~m}$ & $2358.4 \mathrm{~m}$ \\
$\mathbf{1 0}$ & $31.31 \mathrm{~m}$ & $281.83 \mathrm{~m}$ & $1127.32 \mathrm{~m}$ & $2536.47 \mathrm{~m}$ & $4509.12 \mathrm{~m}$ \\
\hline
\end{tabular}

Table 4: The transmission range for different amount of $\lambda$.

Table 4 shows the value of the transmission range at different frequencies by using the Friis free space, Eq. (17). According to the results we can achieve a line of sight transmission range equal to $4.5 \mathrm{~km}$ at $18.75 \mathrm{MHz}$.

To sum up, the study compare the system throughput with TEL100M-C, which is one of the products of the BIOPAC ${ }^{\circledR}$ Systems Corporation. According to the production's information this device is able to achieve an SNR of $50 \mathrm{~dB}$ (nominal) for $0.05-35 \mathrm{~Hz}$ and $40 \mathrm{~dB}$ (nominal) for $0.05-500$ $\mathrm{Hz}$ and transmission range equal to 150 meter line of sight. In addition this device works in the UHF band. Table 2, Table 3 and Fig. 10 show our simulation output results for EEG signals from $0.3 \mathrm{~Hz}$ to $100 \mathrm{~Hz}$. As can be seen for $\mathrm{N}$ equal to ten bits per sample and $\lambda=1$ meter, we achieve a 
transmission range equal to 281 meter which is approximately 2 times larger than the transmission range (line on sight) of this device. For $\lambda$ greater than one meter the results is considerably higher than for this product. According to table 3 the achieved SNR by the proposed method, by utilizing QPSK modulation, is in the $[42 \mathrm{~dB}$, $72 \mathrm{~dB}$ ] interval which is much higher than the achieved SNR by this device.

\section{Conclusion}

Advancement of wireless technology has made some developments in current wireless EEG. For instance, by improving transmission systems for brainwave signals, more opportunities for discovering other aspects of brainwaves have appeared, and patients will benefit from these devices. What has been done in this project is to take advantage of the nature of brainwaves to design a quantizer. Such quantizers have better performance with respect to other scalar quantizers in dealing with brainwave signals. Our experiment has been done with uniform and Gaussian distribution of quantization level and BUQAB and BGQAB have been introduced. They have been compared with other quantization schemes in wireless EEG devices. Furthermore we examined them in a simulated wireless system. Achieved results show significant improvement in the quantization parameter and other related system parameters such as SNR, BER and transmission range.

\section{References}

1. Sanei S, Chambers JA. EEG Signal Processing, 1. ed. Wiley Interscience: New York. 2007. http://dx.doi.org/10.1002/9780470511923

2. Michel CM, Murray MM, Lantz G, Gonzalez S, Spinelli L, Grave de Peralta R. EEG source imaging. Clin. Neurophysiol. 2004;115(10):2195-2222. http://dx.doi.org/10.1016/j.clinph.2004.06.001

3. Argyropoulos S, Tzovaras D, Ioannidis D, Damousis Y, Braun $\mathrm{M}$, Boverie S, et al. Biometric template security in multimodal biometric systems based on error correcting codes. Journal of Computer Securit. 2010;18(1):161-185.

4. Scher MS. Automated EEG-sleep analyses and neonatal neurointensive care. Sleep Medicine. 2004;5(6):533-40. http://dx.doi.org/10.1016/j.sleep.2004.07.002

5. Abaya EF, Wise GL. On the Existence of optimal quantizers. IEEE Trans. Information Theory. 1982;28(6);937-940. http://dx.doi.org/10.1109/TIT.1982.1056582

6. Tolbert JR, Kabali P, Brar S, Mukhopadhyay S. A low power system with adaptive data compression for wireless monitoring of physiological signals and its application to wireless electroencephalograph.11. International Symposium on Quality Electronic Design (ISQED), San Jose, CA. USA. 2010:333-341.
7. Yates DC. A key power trade-off in wireless EEG headset design. IEEE EMBS Conference on Neural Engineering. 2007:453-456. http://dx.doi.org/10.1109/CNE.2007.369707

8. Wang A, Sodini C. A simple energy model for wireless micro sensor transceivers. IEEE Global Telecomm Conf., Texas, USA. 2005:3205-3209.

9. Lin CT, Chen YC, Huang Y, Chiu TT, Ko LW, Liang SF, et al. Development of wireless brain computer interface with embedded multitask scheduling and its application on realtime driver's drowsiness detection and warning. IEEE Trans. Biomed. Eng. 2008;55(5):1582-1591. http://dx.doi.org/10.1109/TBME.2008.918566

10. Sayood K. Introduction to data compression. 3. ed. Elsevier: NewYork. 2006.

11. Bao X, Wang J, Hu J. Method of individual identification based on electroencephalogram analysis. International Conference on New Trends in Information and Service Science. 2009:390-393.

12. Jayant N. Adaptive quantization with one word memory. The Journal of the Acoustical Society of America.1973;54(3):340. http://dx.doi.org/10.1121/1.1978381

13. Mitra D. Mathematical analysis of an adaptive quantizer. Bell Systems Technical Journal. 1974, 53(5), 867-898. http://dx.doi.org/10.1002/j.1538-7305.1974.tb02774.x

14. Sayood K, Gibson JD. Explicit additive noise models for uniform and nonuniform MMSE quantization. Signal Processing Journal. 1984;7(4):407-414. http://dx.doi.org/10.1016/0165-1684(84)90038-0

15. Sayood K, Na S. Recursively indexed quantization of memoryless sources. IEEE Transactions on Information Theory. 1996;38(5):1602-1609. http://dx.doi.org/10.1109/18.149516

16. Farvardin N, Modestino JW. Optimum quantizer Performance for a class of non-Gaussian memoryless sources, IEEE Transactions on Information Theory. 1984;30(3):485-497. http://dx.doi.org/10.1109/TIT.1984.1056920

17. Julius OS. Mathematics of the discrete Fourier Transform (DFT). 2. ed. W3K Publishing.2007.

18. Glover IA, Grant PM. Digital Communications. New Jersey: Prentice Hall. 1998.

19. Abdullah MK, Subari KS, Loong JLCH, Ahmad NN. Analysis of the EEG signal for a practical biometric system. World Academy of Science, Engineering and Technology. 2010;(68):1133-1137.

20. Chi M, Wang Y, Maier C, Jung TP, Cauwenberghs G. Dry and noncontact EEG sensors for mobile brain-computer interfaces. IEEE Trans. Neural Systems and Rehab. Eng. 2012;(20):228-235. http://dx.doi.org/10.1109/TNSRE.2011.2174652

21. Zhang Z, Jung TP. Compressed sensing of EEG for wireless telemonitoring with low energy consumption and inexpensive hardware. IEEE Trans. Biomed. Eng. 2013;60(1):221-224. http://dx.doi.org/10.1109/TBME.2012.2217959 
22. Callan D, Gamez M, Cassel D, Terzibas C, Callan A, Kawato $\mathrm{M}$, et al.Dynamic visuomotor transformation involved with remote flying of a plane utilizes the 'Mirror Neuron' system. PLoS One. 2012;7:1-14.

http://dx.doi.org/10.1371/journal.pone.0033873

23. Marcel S, Millan J. Person authentication using brainwaves (EEG) and maximum a posteriori model adaptation. IEEE Trans. Pattern Analysis and Machine Intelligence. 2012;29(4):743-750.

http://dx.doi.org/10.1109/TPAMI.2007.1012

24. Palaniappan R, Mandic DP. Biometrics from brain electrical activity. A machine learning approach. IEEE Trans. Pattern Analysis and Machine Intelligence. 2007;29:738-742.

http://dx.doi.org/10.1109/TPAMI.2007.1013
25. Palaniappan R,Mandic DP. EEG based biometric framework for automatic identity verification. Journal of VLSI Signal Processing Systems. 2007;49(2):243-250.

26. Usakli AB. Improvement of EEG signal acquisition: An Electrical Aspect for State of the Art of Front End. Computational Intelligence and Neuroscience. 2010; 630649, 7 pages. http://dx.doi.org/10.1155/2010/630649

27. Marks RJ. Introduction to Shannon Sampling and Interpolation Theory, Spinger-Verlag. 1991. http://dx.doi.org/10.1007/978-1-4613-9708-3

28. Tong S, Bezerianos A, Paul J, Zhu Y, Thakor N. Nonextensive entropy measure of EEG following brain injury from cardiac arrest. Journal of Statistical Mechanics and its Applications.2002;305(15):619-625. http://dx.doi.org/10.1016/S0378-4371(01)00621-5 